\title{
Eu-Doped Cerium Oxide Nanoparticles Studied by Positron Annihilation
}

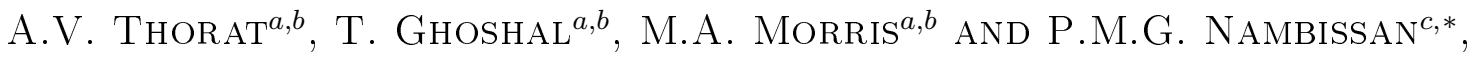 \\ ${ }^{a}$ Materials Research Group, Department of Chemistry and Tyndall National Institute \\ University College Cork, Cork, Ireland \\ ${ }^{b}$ Centre for Research on Adaptive Nanostructures and Nanodevices, Trinity College Dublin, Dublin, Ireland \\ ${ }^{c}$ Applied Nuclear Physics Division, Saha Institute of Nuclear Physics, Kolkata 700064, India
}

\begin{abstract}
The defect characteristics of cerium oxide $\left(\mathrm{CeO}_{2}\right)$ nanoparticles prepared through a solvothermal process and doped by europium to different concentrations ( $[\mathrm{Eu}]=0,0.1,0.5,1, \ldots, 50 \mathrm{wt} \%$ ) were studied by positron lifetime and coincidence Doppler broadening measurements. The particle sizes estimated from X-ray diffraction showed a reducing trend with increasing doping concentration except an increase during $[\mathrm{Eu}]=0.1-1 \mathrm{wt} \%$. The latter effect is attributed to the reduction of $\mathrm{Ce}^{4+}$ ions to $\mathrm{Ce}^{3+}$ resulting into the release of vacancies and formation of $\mathrm{Ce}^{3+}-$ -vacancy associates. The lattice parameter increased with the decrease in particle size. Quantum confinement effects were observed in optical absorption studies as increase of band gap in particles of sizes below 7-8 nm. The vacancy-type defects were investigated by positrons. A lifetime of $176 \pm 4$ ps less than 187-189 ps reported for positrons in bulk $\mathrm{CeO}_{2}$ reveals trapping of positrons in vacancy-type defects within the nanocrystallites. The defect-specific positron lifetime is admixed with that at the crystallite surfaces and it increased due to vacancy agglomeration at higher doping concentrations. Coincidence Doppler broadening studies indicated positron annihilation in defects surrounded by oxygen ions and the $S-W$ plot showed the effect of quantum confinement through a peak-like kink or shoulder in the plot. Optical absorption studies have supported this observation.
\end{abstract}

DOI: 10.12693 /APhysPolA.125.756

PACS: 61.46.Df, 78.20.Ci, 61.72.jd, 12.38.Aw, 78.70.Bj

\section{Introduction}

The defect characteristics of semiconductor nanocrystalline materials are of foremost relevance when they are considered for applications in science and technology, the reason being rather simple that their properties are overwhelmingly modified, if not decided, by the defects formed in various forms and at various sites within the lattice $[1,2]$. Not surprisingly, there is an increasing interest in investigating their behaviour under different physical conditions using diverse experimental techniques. In this work, we have chosen nanoparticulate cerium oxide $\left(\mathrm{CeO}_{2}\right)$ doped by europium $([\mathrm{Eu}]=0,0.1$, $0.5, \ldots, 50 \mathrm{wt} \%)$ as a potential case for investigation for their defects and defect-related aspects by, among other techniques, positron lifetime and coincidence Doppler broadening spectroscopic (CDBS) measurements. The utility of positron annihilation spectroscopy for this kind of studies is by now well established and review articles on the subject are available in literature $[3,4]$.

\section{Experimental details}

The samples were prepared through a solvothermal process at $180^{\circ} \mathrm{C}$ for $12 \mathrm{~h}$ using $\mathrm{Ce}\left(\mathrm{NO}_{3}\right)_{3} \cdot 6 \mathrm{H}_{2} \mathrm{O}$, ammonia and $\mathrm{Eu}\left(\mathrm{NO}_{3}\right)_{3} \cdot 5 \mathrm{H}_{2} \mathrm{O}$ as essential reagents. The precipitates in fine powder form were collected, washed

*corresponding author; e-mail: pmg.nambissan@saha.ac.in several times with deionized water and absolute ethanol and then dried at $50^{\circ} \mathrm{C}$ in air.

Positron lifetime and CDBS measurements were carried out by immersing into the volume of the powder a $0.4 \mathrm{MBq}$ strong ${ }^{22} \mathrm{Na}$ positron source taken in the form of deposition of the ${ }^{22} \mathrm{NaHCO}_{3}$ (iThemba Labs, SA) salt on to a $2 \mathrm{mg} \mathrm{cm}^{-2}$ thick Ni foil. The salt-deposited foil, after drying of the solvent, had been folded along a line $0.5 \mathrm{~mm}$ away from the salt-deposited area to conceal the source from coming in direct contact with the sample material. Prior to the deposition of the salt, the Ni foil had been annealed at $1000^{\circ} \mathrm{C}$ for $2 \mathrm{~h}$ in vacuum $\left(\approx 10^{-5} \mathrm{mbar}\right)$ and slowly cooled to ensure that there existed no vacancies or dislocations which may trap positrons while penetrating through it. The positron lifetime spectra recorded using this source in sandwich geometry with high pure $(99.999 \%)$, single crystalline and well-annealed Si samples had reproduced its bulk lifetime of 220 ps, indicating that the rest of the components originated from the source material and the foil. "Source correction" has been done to remove these contributions when the spectra were analysed using PALSfit [5].

The positron lifetime spectra were recorded using a slow-fast coincidence spectrometer with resolution $170 \mathrm{ps}$ and about $1 \times 10^{6}$ coincidence counts were acquired under each spectrum. The CDBS measurements were carried out using two high pure Ge detectors of energy resolution $1.27 \mathrm{keV}$ and $1.33 \mathrm{keV}$ at $511 \mathrm{keV}$ and kept on either side of the source-sample assembly facing each other. The data to yield about $8 \times 10^{6}$ coincidence events under the $2 d$-spectrum were acquired and analysed using the 
software LAMPS developed by TIFR-BARC Pelletron Group, Mumbai [6]. During the measurements, the glass tube containing the sample and the source had been continuously evacuated to $p=10^{-3}$ mbar to get rid of air or absorbed gases and keep them in dry conditions.

\section{Results and discussion}

The samples were characterized for the purity of phase by X-ray diffraction (Fig. 1). The patterns exhibited peaks which could be indexed to a cubic fluorite phase of ceria. The particle sizes were estimated from peak width analysis and using Scherrer equation [7] and they decreased with increasing Eu concentration although interspersed by an increase during $[\mathrm{Eu}]=0.1-1 \mathrm{wt} \%$ (not shown). The decrease is caused by the relaxation of the strain induced by the substitution of $\mathrm{Ce}^{4+}$ ions of radius $0.97 \AA$ by $\mathrm{Eu}^{3+}$ ions of larger radius $1.066 \AA$. But the reduction of $\mathrm{Ce}^{4+}$ to $\mathrm{Ce}^{3+}$ facilitated substitution free of strain as the ionic radius of $\mathrm{Ce}^{3+}$ is $1.143 \AA$. The reduction results in the release of $\mathrm{O}^{2-}$ vacancies and formation of $\mathrm{Ce}^{3+}$-vacancy associates that have resulted into the intermediate increase in particle size. The lattice parameter increased with the decrease in particle size and decreased when the particle sizes increased in the intermediate range of doping. This resulted into a linear relation between the two physical parameters when plotted in a log-log scale [8]. Further, optical absorption measurements had shown quantum confinement effects which manifested in the form of increase of band gap in particles of sizes below $7-8 \mathrm{~nm}[8]$.

The vacancy-type defects were investigated using positrons. The positron lifetime spectra gave three lifetimes. The shortest one $\tau_{1}=176 \pm 4 \mathrm{ps}$ obtained for all the samples was less than 187-189 ps reported for positron annihilation in bulk $\mathrm{CeO}_{2}[9,10]$, indicating positron trapping in vacancy-type defects within the nanocrystallites. The variation of the defect-specific lifetime $\tau_{2}$ during the increase in concentration of doping is shown in Fig. 2. The very large values of $\tau_{2}$ suggest that it is an admixture of two contributions, one from the vacancy-type defects within the crystallites and the other from the surfaces. The latter comes as a result of a fraction of positrons thermally diffusing out to the crystallite surfaces before annihilation takes place. Although Uedono et al. [11] have reported a thermal diffusion length of $2.7 \pm 0.5 \mathrm{~nm}$ for epitaxially grown $\mathrm{CeO}_{2}$ films, it is likely to be somewhat larger in the powdered nanoparticulate samples considered here. Initially a number of existing vacancies get filled by the dopant ions and hence this contribution to $\tau_{2}$ gets reduced. The dominance of the surface contribution will then tilt it towards higher values, as observed between $[\mathrm{Eu}]=0-0.1 \mathrm{wt} \%$. The succeeding fall of $\tau_{2}$ accompanying the intermediate increase in particle size during $[\mathrm{Eu}]=0.1-1 \mathrm{wt} \%$ appears to result from the release of oxygen vacancies and the formation of vacancy associates with the $\mathrm{Ce}^{3+}$ ions [12]. All the positron lifetimes $\tau_{1}, \tau_{2}$ and $\tau_{3}$ decreased whereas

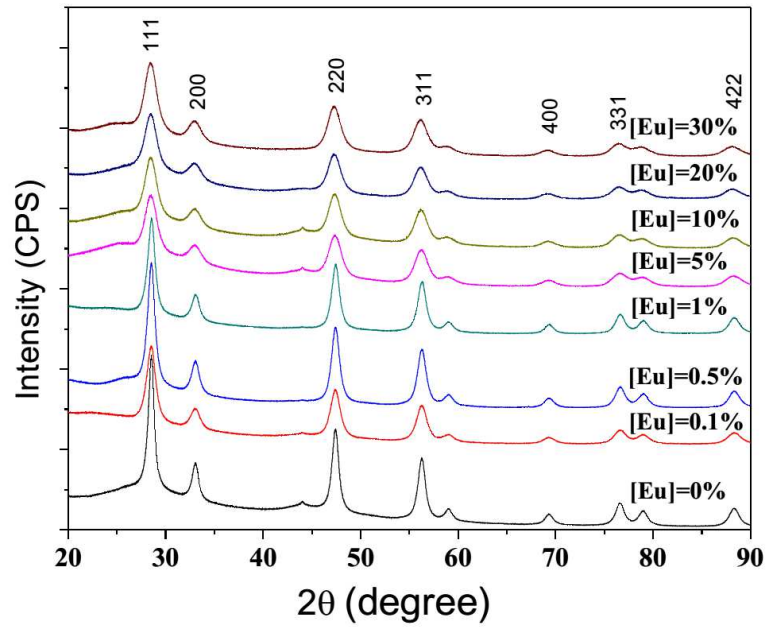

Fig. 1. X-ray diffraction patterns of the undoped and some of the Eu-doped cerium oxide nanocrystalline samples.

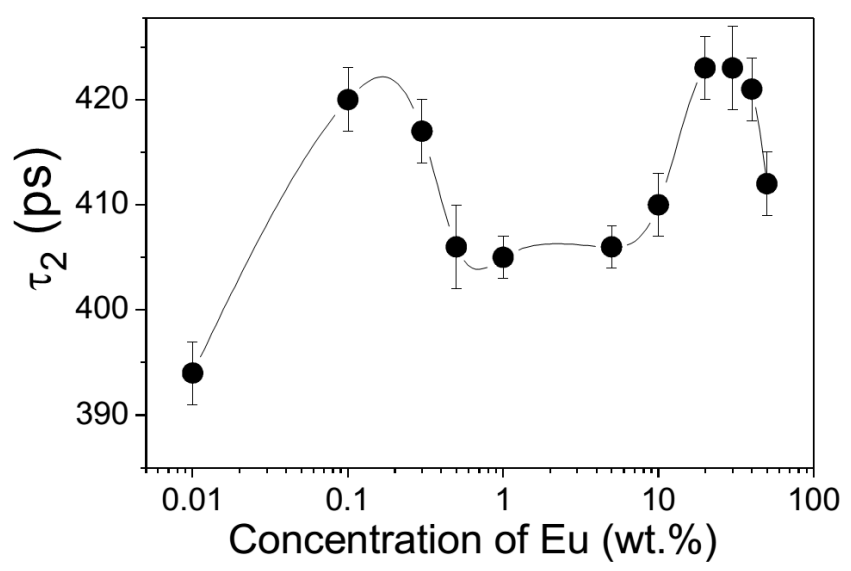

Fig. 2. The intermediate positron lifetime $\tau_{2}$ versus the concentration of $\mathrm{Eu}$ in the cerium oxide nanoparticulate samples.

the relative intensities $I_{2}$ and $I_{3}$ increased during this process. The increase in the intensities especially in $I_{2}$ (not shown) confirms the presence of additional vacancy-type defects at this stage in the materials. As already mentioned, since $\tau_{2}$ is a weighted average of the two lifetimes, its value will tilt towards that in the species of traps which dominates at the particular stage. Hence the reduction of $\tau_{2}$ proves the generation of excess vacancy clusters within the nanocrystallites, wherein the positron lifetimes are expected to be in the range 277-293 ps [8], due to the reduction of $\mathrm{Ce}^{4+}$ to $\mathrm{Ce}^{3+}$ ions. Subsequently thereafter, the vacancy-type defects agglomerate to give larger positron lifetimes, as seen from $[\mathrm{Eu}]=1-20 \mathrm{wt} \%$, and beyond this, $\tau_{2}$ falls due to the excess unincorporated $\mathrm{Eu}^{3+}$ ions.

The longest component $\tau_{3}$ and its intensity $I_{3}$ arise from ortho-positronium (o-Ps) atoms associated with the high degree of porosity of the samples and also those 

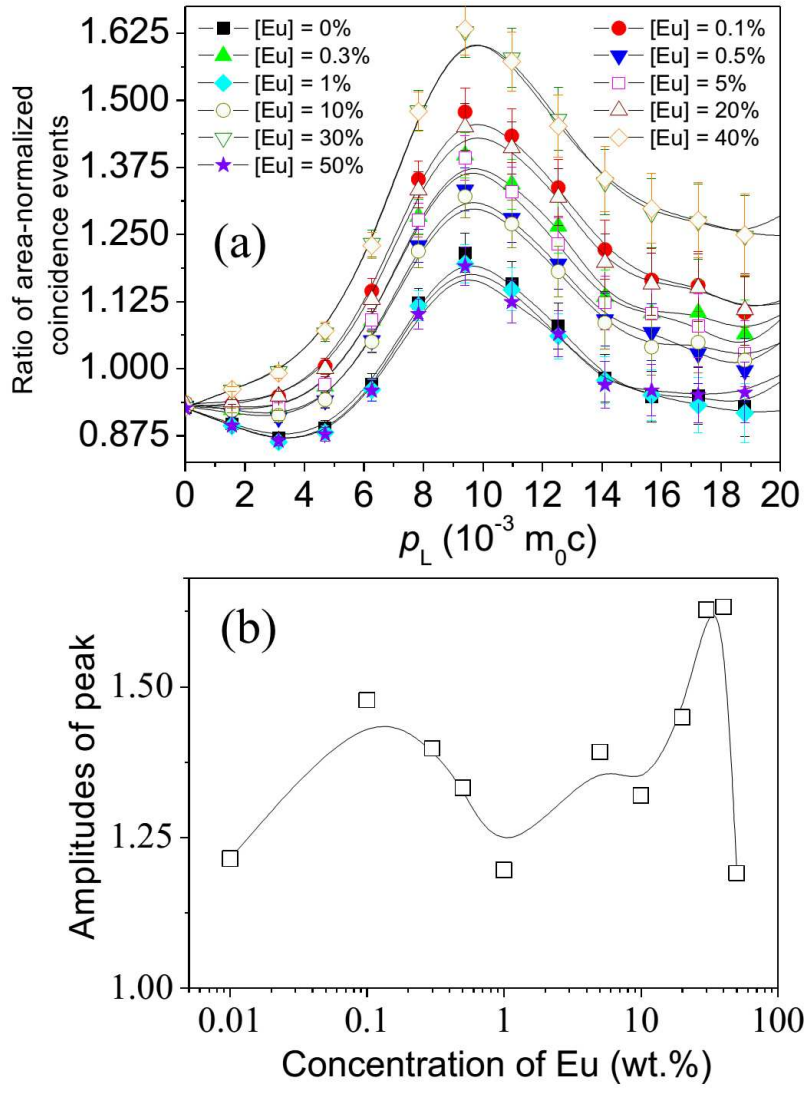

Fig. 3. (a) Ratio curves generated from the CDB spectra with respect to Al. (b) Amplitudes of the peak at $p_{\mathrm{L}}=9.4 \times 10^{-3} m_{0} c$ of the ratio curves in Fig. 3a.

formed in the intercrystallite regions. Their variations have been discussed elsewhere [8].

The results of CDBS, as given in Fig. 3a in the form of ratio curves generated with respect to high pure (99.999\%) single crystalline $\mathrm{Al}$, show a prominent peak appearing at $p_{\mathrm{L}}=9.4 \times 10^{-3} m_{0} c$ which arises from the $2 p$ electrons of oxygen. The spectra had been normalized for same area within a momentum spread of $p_{\mathrm{L}}= \pm 50 \times 10^{-3} m_{0} c$ on either side of the peak of the actual experimental spectra and the full ratio curves also included a number of other peaks which are not discussed here [8]. The amplitude of this peak with Eu concentration in the samples shows a characteristic decrease during $[\mathrm{Eu}]=0.1$ to $1 \mathrm{wt} \%$ (Fig. 3b). Remarkably, this is the range of concentrations where almost all parameters like particle size, lattice constant, and positron lifetimes measured from different experimental methods have shown abrupt changes from the prevailing trend and it signifies the formation of $\mathrm{Ce}^{3+}$-vacancy associates prompted by the release of oxygen vacancies due to the reduction of Ce ions from their higher ionized state to the lower one. It thus emphasizes the initial trapping of a fraction of positrons in vacancy-type defects within the nanocrystallites, as indicated by the results of positron lifetimes
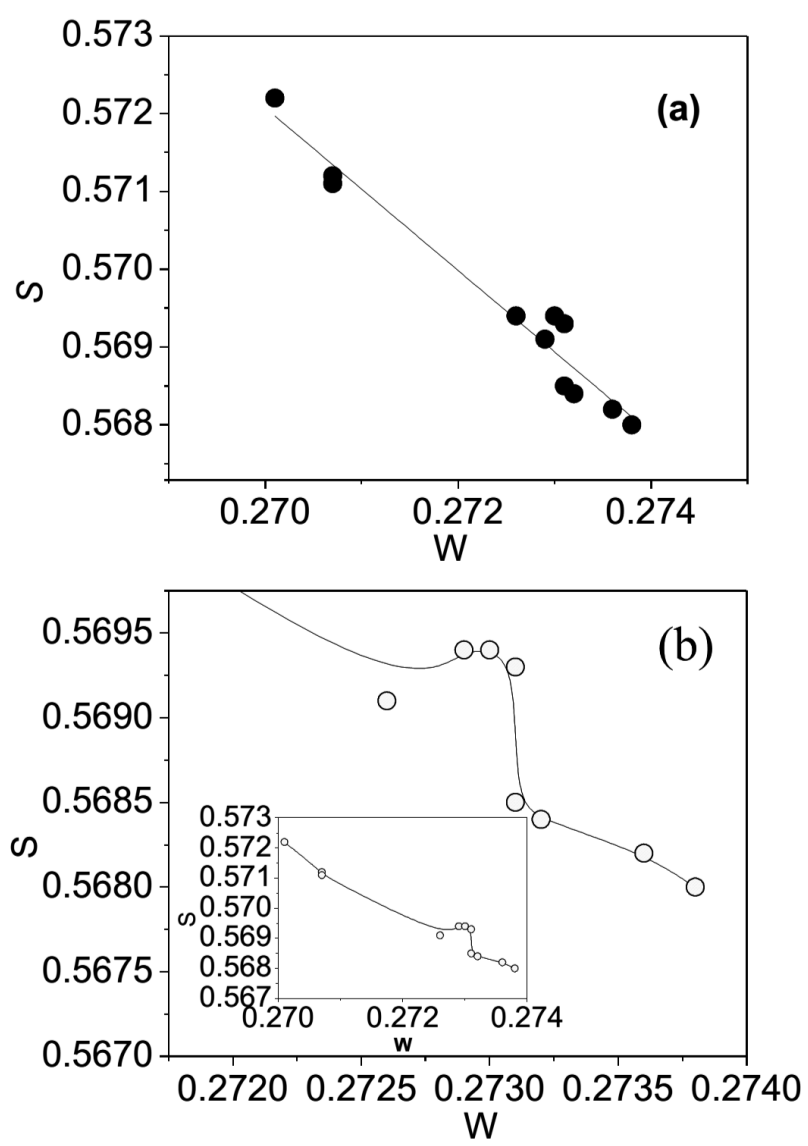

Fig. 4. (a) The $S-W$ plot of Eu-doped $\mathrm{CeO}_{2}$ nanoparticles with increasing concentration of Eu. (b) The $S-W$ plot of Eu-doped $\mathrm{CeO}_{2}$ nanoparticles with increasing particle size. Inset shows the full plot.

as well. Yet another analysis performed in this study is the derivation of the lineshape parameters $S$ and $W$ according to the usual definitions and a look at their variations supports the arguments made above related to vacancy aggregation at higher concentrations of doping. The $S-W$ plot expectedly is a straight line (Fig. 4a).

However, a change of the leading parameter from the concentration of doped $\mathrm{Eu}$ ions to the particle sizes gives another interesting feature. It gives a peak-like kink or shoulder indicating phenomena other than defect evolution or defect interaction at this stage (Fig. 4b). This has happened at around the same particle size below which a characteristic blue shift had been observed in the optical absorption spectra and a corresponding increase in the band gap energy [8]. A deterministic process that can bring in a changed electronic density and momentum distribution is the quantum confinement effect where the reduced probability of annihilation of positrons with core electrons results in a conspicuous rise of the $S$ parameter. In a way it proves that positron annihilation as a technique is reliable for better understanding of the size-induced changes and defects-related aspects in nanocrystalline systems. 


\section{Summary and conclusions}

The doping by europium has caused a number of changes in nanoparticles of cerium oxide. The sizes of the nanoparticles significantly got reduced with the increase of concentration of Eu, except in a sensitive range from 0.1 to $1 \mathrm{wt} \%$ which was identified as a stage where cerium ions are reduced in their oxidation states and oxygen vacancies are released in the sample. These vacancies form complexes with the reduced $\mathrm{Ce}$ ions. $\mathrm{Ce}^{3+}$ ions have a higher radius compared to $\mathrm{Ce}^{4+}$ and hence the vacancies released by them are of larger size. The occupancy of the same by $\mathrm{Eu}^{3+}$ ions of comparable radius does not introduce strain and the lattice parameter has shown a decrease at this stage, notwithstanding its overall increase. At higher concentrations of doping, vacancy aggregation is observed. CDBS results also supported these findings by exhibiting well defined variations of the amplitude of the prominent oxygen $2 p$ electron peak. In particular, optical absorption has shown widening of the band gap due to quantum confinement effects and positron annihilation also showed the corresponding changes in the form of changes in positron lifetimes and lineshape parameters.

\section{References}

[1] L. Chen, P. Fleming, V. Morris, J.D. Holmes, M.A. Morris, J. Phys. Chem. C 114, 12909 (2010).

[2] S. Tsunekawa, J.-T. Wang, Y. Kawazoe, J. Alloys Comp. 408-412, 1145 (2006).

[3] P.M.G. Nambissan, in: Nanotechnology: Synthesis and Characterization, Vol. 2, Eds. Shishir Sinha, N.K. Navani, J.N. Govil, Studium Press LLC, Houston 2013 , p. 455.

[4] P.M.G. Nambissan, J. Phys., Conf. Series 443 , 012040 (2013).

[5] J.V. Olsen, P. Kirkegaard, N.J. Pedersen, M. Eldrup, Phys. Status Solidi C 4, 4004 (2007).

[6] A. Chatterjee, K. Ramachandran, A. Kumar, A. Behere, Linux Advanced Multi Parameter System (2013).

[7] A.L. Patterson, Phys. Rev. 56, 978 (1939).

[8] A.V. Thorat, T. Ghoshal, J. Holmes, P.M.G. Nambissan, M.A. Morris, Nanoscale 6, 608 (2014).

[9] S.J. Chang, M. Li, Q. Hua, L.J. Zhang, Y.S. Ma, B.J. Ye, W.X. Huang, J. Catalysis 293, 195 (2012).

[10] A. Sachdeva, S.V. Chavan, A. Goswami, A.K. Tyagi, P.K. Pujari, J. Solid State Chem. 178, 2062 (2005).

[11] A. Uedono, K. Shimoyama, M. Kiyohara, K. Yamabe, J. Appl. Phys. 94, 5193 (2003).

[12] S. Deshpande, S. Patil, S.V.N.T. Kuchibhatla, S. Seal, Appl. Phys. Lett. 87, 133113 (2005). 